Case Report

\title{
A CASE REPORT ON STEVENS-JOHNSON SYNDROME
}

\author{
Babu Dharmarajan ${ }^{1}$, Sanil Varghese ${ }^{2} \&$ Sachina B.T. ${ }^{3}$ \\ ${ }^{1}$ Associate Professor \& HOD, ${ }^{2,3}$ Lecturers, Department of M edical Surgical Nursing, Yenepoya Nursing College, \\ Yenepoya University, M angalore - 575 018, India. \\ Correspondence : \\ Babu Dharmarajan \\ Associate Professor, HOD, Department of M edical Surgical Nursing, Yenepoya Nursing College, \\ Yenepoya University, University Road, M angalore - 575 018, India. \\ E-mail : babu_dharma2007@ rediffmail.com
}

\section{Abstract:}

Stevens-J ohnson syndrome (SSS) and toxic epidermal necrolysis (TEN) are a rare disease which is considered to be more devastating and fatal in nature. Usually symptoms may start with the involvement of epidermis and progress to the various manifestations. Early findings, symptomatic and supportive treatment will have the more positive impact on the outcome of the client.

Keywords: Toxic epidermal necrolysis, skin lesion, Erythematous macules

\section{Introduction:}

31 year old lady admitted to the hospital with the chief complaints of high fever, chills, fatigue, sore throat, and edema over the face, lesions over the body and resembled to be affected with burns. On detailed history it was evidenced that she was admitted to some local hospital for the treatment of fever, during the treatment she started developing edema, lesions over the entire body; On physical examination the patient is semiconscious, poorly built, skins are peeling out, (Fig 1) whitish patches in cornea, external ear skin peals out, rhinorrea, reddish lips and bleeding, rashes over the abdomen (Fig 2) and restricted movements were evident. Blood investigation evidenced the decreased level of hemoglobin and platelet counts; culture and hypersensitivity to amikacin, penicllin, cifrofloxacin, ciftriaxone and found to be allergic to ciprofloxacin. The diagnosis was confirmed as Stevens Johnson Syndrome/Toxic epidermal necrolysis. The patient was treated with Metronidazole $1 \%$ gel, paracetamol, pantoprazole and candid. There was a significant

\begin{tabular}{|c|}
\hline Access this article online \\
\hline Quick Response Code \\
\hline
\end{tabular}

improvement was seen in the patient.

\section{Discussion :}

Stevens - Johnson syndrome (SSS) and toxic epidermal necrolysis (TEN) are severe adverse reactions to medication. SJS and TEN are conditions characterized by erythematous macules evolving to epidermal detachment and mucous membrane erosions. In SJ Sthere is less than $10 \%$ body surface area involvement, in TEN more than $30 \%$ and $10-30 \%$ overlap cases are evident ${ }^{1}$.

TEN and SJS affecting approximately 1or 2/1,000,000 annually, and are considered medical emergencies as they are potentially fatal. Drugs are assumed or identified as the main cause of SJS/TEN in most cases, but Mycoplasma pneumoniae and Herpes simplex virus infections are well documented causes alongside rare cases in which the aetiology remains unknown. Several drugs are at "high" risk of inducing TEN/SJS viz., Allopurinol, Trimethoprimsulfamethoxazole and other sulfonamide-antibiotics, aminopenicillins, cephalosporins, quinolones, carbamazepine, phenytoin, phenobarbital and NSAID's of the oxicam-type?

Treatment is primarily supportive and symptomatic. Patients should be treated with special attention to airway and hemodynamic stability, fluid status, wound/burn care, and pain control. Prime care must be directed to fluid replacement and electrolyte correction. Few physicians advocate corticosteroids, cyclophosphamide, plasmapheresis, hemodialysis, and immunoglobulin and oral lesions can be well managed with mouthwashes whereas topical anesthetics are useful in reducing pain and 
allowing the patient to take fluids. Skin lesions should be treated as burns and areas of denuded skin must be covered with compresses of saline $e^{3}$. To conclude the treatment and management of SJS/TEN should be based multidisciplinary approach.

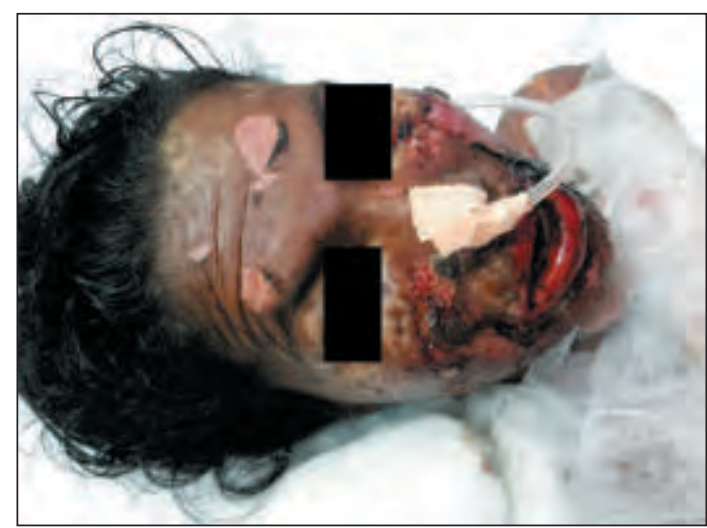

Fig 1: Showing skin peeling over the face and hemorrhagic oral mucosa

\section{References:}

1. HHF Ho, Diagnosis and Management of Stevens-Johnson Syndrome and Toxic Epidermal Necrolysis. The Hong Kong M edical Diary. VOL.13 NO.10 OCTOBER 2008, Pp:17

2. French E Lars, Harr Thomas. Toxic epidermal necrolysis and StevensJohnson syndrome. OrphanetJ Rare Dis. 2010; 5: 39

3. Stephen Foster C, Rola Ba-Abbad. Stevens-Johnson Syndrome Treatment \& M anagement. M edscape.

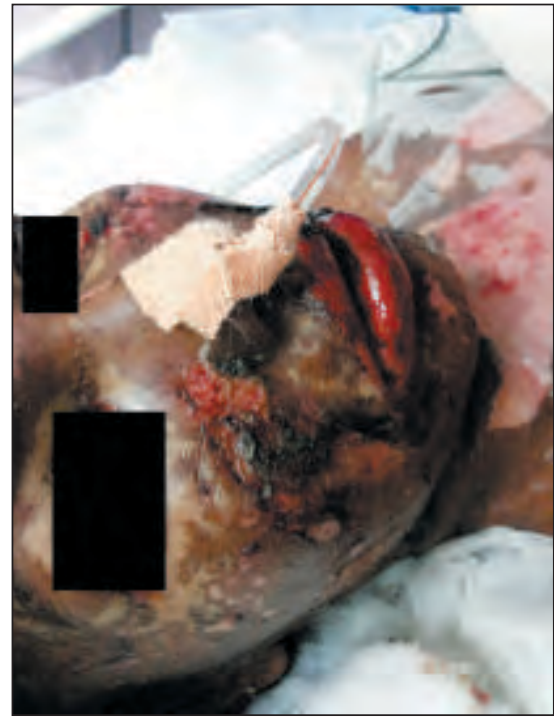

Fig 2 : Blisters and red splotches on the face, mouth and neck 